\title{
TEMPERATURE DISTRIBUTION OF A NON-FLARING ACTIVE REGION FROM SIMULTANEOUS HINODE XRT AND EIS OBSERVATIONS
}

\author{
Paola Testa ${ }^{1}$, Fabio Reale ${ }^{2,3}$, Enrico Landi ${ }^{4}$, Edward E. DeLuca ${ }^{1}$, And Vinay KashyaP ${ }^{1}$ \\ ${ }^{1}$ Smithsonian Astrophysical Observatory, 60 Garden Street, MS 58, Cambridge, MA 02138, USA; ptesta@cfa.harvard.edu \\ 2 Dipartimento di Scienze Fisiche ed Astronomiche, Sezione di Astronomia, Università di Palermo, Piazza del Parlamento 1, 90134, Italy \\ ${ }^{3}$ INAF-Osservatorio Astronomico di Palermo, Piazza del Parlamento 1, 90134 Palermo, Italy \\ ${ }^{4}$ Department of Atmospheric, Oceanic and Space Sciences, University of Michigan 2455 Hayward St., Ann Arbor, MI 48109, USA \\ Received 2010 June 21; accepted 2010 November 27; published 2011 January 17
}

\begin{abstract}
We analyze coordinated Hinode X-ray Telescope (XRT) and Extreme Ultraviolet Imaging Spectrometer (EIS) observations of a non-flaring active region to investigate the thermal properties of coronal plasma taking advantage of the complementary diagnostics provided by the two instruments. In particular, we want to explore the presence of hot plasma in non-flaring regions. Independent temperature analyses from the XRT multi-filter data set, and the EIS spectra, including the instrument entire wavelength range, provide a cross-check of the different temperature diagnostics techniques applicable to broadband and spectral data, respectively, and insights into cross-calibration of the two instruments. The emission measure distributions, $(\operatorname{EM}(T))$, we derive from the two data sets have similar width and peak temperature, but show a systematic shift of the absolute values, the $\operatorname{EIS}(\operatorname{EM}(T))$ being smaller than the XRT $(\operatorname{EM}(T))$ by approximately a factor two. We explore possible causes of this discrepancy, and we discuss the influence of the assumptions for the plasma element abundances. Specifically, we find that the disagreement between the results from the two instruments is significantly mitigated by assuming chemical composition closer to the solar photospheric composition rather than the often adopted "coronal" composition. We find that the data do not provide conclusive evidence on the high temperature $(\log T(\mathrm{~K}) \gtrsim 6.5)$ tail of the plasma temperature distribution, however, suggesting its presence to a level in agreement with recent findings for other non-flaring regions.
\end{abstract}

Key words: Sun: abundances - Sun: activity - Sun: corona - Sun: UV radiation - Sun: X-rays, gamma rays techniques: spectroscopic

\section{INTRODUCTION}

Understanding how solar and stellar coronae are heated to high temperatures is one of the most important open issues in astrophysics. Coronal heating is clearly related to the strong magnetic fields that fill the atmospheres of the Sun and solarlike stars, but the mechanism that converts magnetic energy into thermal energy remains unknown. Theoretical models based on steady heating failed to explain the physical properties observed in coronal structures. A promising and widely studied framework for understanding coronal heating is the nanoflare model proposed by Parker (1972). In this model, convective motions in the photosphere lead to the twisting and braiding of coronal magnetic field lines. This topological complexity ultimately leads to the formation of current sheets, where the magnetic field can be rearranged through the process of magnetic reconnection. This model has been further refined and adapted to a scenario where nanoflares occur in unresolved strands in coronal loops below the spatial resolution of available instrumentation (Parker 1988; Cargill 1994; Cargill \& Klimchuk 1997; Klimchuk 2006; Warren et al. 2003; Parenti et al. 2006).

Nanoflare models predict the presence of very hot plasma with temperatures in excess of $3 \mathrm{MK}$ in non-flaring solar regions; depending on the energy of the nanoflare, temperatures may reach or even exceed $10 \mathrm{MK}$. Unambiguous detection of such extreme temperatures in non-flaring solar regions can provide convincing evidence for the presence of nanoflares. However, such detection is not easy, as the amount of very hot plasma produced by nanoflares is expected to be very small. Recent studies have provided some evidence of the presence of hot plasma in the non-flaring Sun. McTiernan (2009) carried out an analysis of the temperature and emission measure determined from quiescent plasma during the 2002-2006 decay phase of solar cycle 23, as derived from GOES and RHESSI observations. He found a persistent faint plasma component with temperatures approximately constant during the entire 2002-2006 interval, and approximately between 5 and $10 \mathrm{MK}$. However, GOES and RHESSI provided different values of such temperatures, and their results were not necessarily well correlated; also, this analysis relied on the isothermal plasma assumption.

Other studies tried to identify the hot plasma through the Xray emission observed by the X-ray Telescope (XRT; Golub et al. 2007) on board Hinode (Kosugi et al. 2007). Reale et al. carried out a temperature analysis of a non-flaring active region, first with only XRT multi-filter data (Reale et al. 2009b) and then combining XRT and RHESSI observations (Reale et al. 2009a). Their findings point to the presence of small amounts of very hot plasmas, with temperatures of $\simeq 5-10 \mathrm{MK}$, and emission measure of the order of few percent of the dominant cool component. These characteristics of the emission measure distribution are compatible with the predictions of nanoflare models. However, they spelled out and discussed the main limitations of their study and similar analyses. First, XRT is also sensitive to plasma at normal active region temperatures (2-3 MK) and thus contamination from the colder active region plasma is a considerable obstacle to the detection of the much smaller amounts of hot plasma; also, the limited temperature resolution of XRT prevents a detailed study of the cold component. Second, RHESSI sensitivity makes it very hard to even detect the quiescent active region plasma. Third, instrumental calibration is an issue for both instruments. Schmelz et al. (2009a) also detected a faint hot temperature tail to the emission measure distribution of active region plasma and determined its temperature to be around $30 \mathrm{MK}$. Subsequent 
Table 1

Details of Hinode XRT and EIS Observations Analyzed in This Paper and Shown in Figures 1 and 2

\begin{tabular}{|c|c|c|c|c|c|c|c|}
\hline \multirow{2}{*}{$\begin{array}{l}\text { Observation } \\
\text { Parameters }\end{array}$} & \multicolumn{6}{|c|}{ XRT } & \multirow{2}{*}{$\begin{array}{c}\text { EIS } \\
171-212 \AA, 245-291 \AA\end{array}$} \\
\hline & Al-poly & C-poly & Ti-poly & Be-thin & Be-med & Al-med & \\
\hline FOV & \multicolumn{6}{|c|}{$384^{\prime \prime} \times 384^{\prime \prime}$} & $128^{\prime \prime} \times 128^{\prime \prime}$ \\
\hline START OBS & \multicolumn{6}{|c|}{ 2008-06-20T23:27:30 } & 2008-06-20T23:03:39 \\
\hline END OBS & \multicolumn{6}{|c|}{ 2008-06-21T03:38:50 } & 2008-06-21T02:19:14 \\
\hline$t_{\exp }(\mathrm{s})$ & 4.1 & 8.2 & 8.2 & 23 & 33 & 46 & 90 \\
\hline
\end{tabular}

analyses by Schmelz et al. (2009b) included RHESSI data and, while confirming the presence of such hot material, could not reconcile the XRT and RHESSI observations using the standard calibration of both instruments. A self-consistent solution was only found if a series of instrumental parameters and the plasma element abundances were adjusted, and the temperature of the hot plasma decreased.

Ample efforts have been devoted to the accurate determination of the thermal structuring of coronal plasma to derive robust observational constraints to the mechanism(s) of coronal heating. The plasma temperature distribution of the quiet corona and of active regions has been investigated through imaging data and spectroscopic observations (e.g., Brosius et al. 1996; Landi \& Landini 1998; Aschwanden et al. 2000; Testa et al. 2002; Del Zanna \& Mason 2003; Reale et al. 2007; Landi et al. 2009; Shestov et al. 2010; Sylwester et al. 2010). Several recent works have focused on EUV spectra obtained with the Hinode Extreme Ultraviolet Imaging Spectrometer (EIS; Culhane et al. 2007) which provides good temperature diagnostic capability, together with higher spatial resolution and temporal cadence than previously available (e.g., Watanabe et al. 2007; Warren et al. 2008; Patsourakos \& Klimchuk 2009; Brooks et al. 2009; Warren \& Brooks 2009).

In the present work, we address the issue of determining the temperature distribution of coronal plasma from a different perspective: we investigate thermal properties of coronal plasma in non-flaring active regions using simultaneous Hinode observations with XRT and with EIS, which provide complementary diagnostics for the X-ray emitting plasma. The multi-filter XRT data set together with EIS spectra, including its entire wavelength range, allows us to accurately determine the thermal structure of the active region plasma and to explore the presence of hot plasma in non-flaring regions. We use spectroscopic observations from the Hinode/EIS instrument of a quiescent active region to constrain the emission measure distribution of the bulk of the active region plasma with the spectral lines observed by EIS in the 171-212 $\AA$ and 245-291 A spectral ranges (see also, e.g., Young et al. 2007; Doschek et al. 2007). Since EIS is most sensitive to plasma with temperatures of 0.6-2 MK, EIS spectra allow us to accurately determine the emission measure distribution of the quiescent active region plasma, to evaluate the fraction of the observed XRT count rates that it emits, and thus to investigate the true amount of emission from the nanoflaring plasma. Thus, the combination of XRT and EIS observations of the same active region allows us to characterize the plasma temperature distribution with better detail than in previous studies. In fact, while some previous studies have made use of data from both imaging and spectroscopic data to constrain the properties of the emitting plasma (e.g., Warren et al. 2010; Landi et al. 2010; O’Dwyer et al. 2011), to our knowledge no previous work has carried out a determination of the temperature distribution by combining XRT and EIS data, nor a quantitative comparison of the independent analysis from the different instruments, as we do here. Independent temperature analysis from the two data sets provide a cross-check of the different temperature diagnostics techniques applicable to spectral and broadband data, respectively, and insights into cross-calibration of the two instruments.

The observations are described in Section 2. The data analysis and results of the determination of the plasma temperature distribution are presented in Section 3. Our findings are discussed in Section 4 and summarized in Section 5.

\section{OBSERVATIONS}

We observed the non-flaring NOAA active region 10999 close to disk center, beginning on 2008 June 20 around 23 UT for several hours with both the XRT and the EIS on board Hinode. The details of the observations are presented in Table 1.

XRT observed AR 10999 in several filters, with a field of view (FOV) of $384^{\prime \prime} \times 384^{\prime \prime}$, for about $4 \mathrm{hr}$ starting 2008 June 20 at 23:27 UT, with a cadence of about 5 minutes in each filter. We analyze observations in the following filters: Al-poly, C-poly, Ti-poly, Be-thin, Be-med, and Al-med. The XRT data were processed with the standard routine xrt_prep, available in SolarSoft to remove the CCD dark current, and cosmic-ray hits. Figure 1 shows the images of XRT observations in Al-poly, Bethin, and Be-med, integrated over the entire observing time (see also Table 1 for details).

The FOV of the EIS observations analyzed here is $128^{\prime \prime} \times 128^{\prime \prime}$ and was built up by stepping the $1^{\prime \prime}$ slit from solar west to east over a $3.5 \mathrm{hr}$ period from 23:03 UT of June 20 to 02:19 UT of June 21. The study includes full spectra on both the EIS detectors from 171 to $212 \AA$ and 245 to $291 \AA$. The exposure time was $90 \mathrm{~s}$ and the study acronym is HPW001_FULLCCD_RAST. The observations were carried out during eclipse season and they were not paused during eclipses: the black stripes of missing data in Figure 2 correspond to Hinode eclipses. The EIS data are processed with the eis_prep routine available in SolarSoft to remove the CCD dark current, cosmic-ray strikes on the $\mathrm{CCD}$, and take into account hot, warm, and dusty pixels. In addition, the radiometric calibration is applied to convert the data from photon events to physical units. The EIS routine eis_ccd_offset is then used to correct for the wavelengthdependent relative offset of the two CCDs of 1-2 pixels in the $X$-direction and $\sim 18$ pixels in the $Y$-direction. Figure 2 shows images obtained from EIS observations by integrating over narrow wavelength ranges each dominated by a single line with different characteristic temperature of formation. We also show three small areas of the active region which have been selected for the detailed analysis of thermal structuring (see the next section for details).

\section{ANALYSIS METHODS AND RESULTS}

Inspection of the XRT observations, in all filters, indicate that the active region is characterized by a modest level of variability 

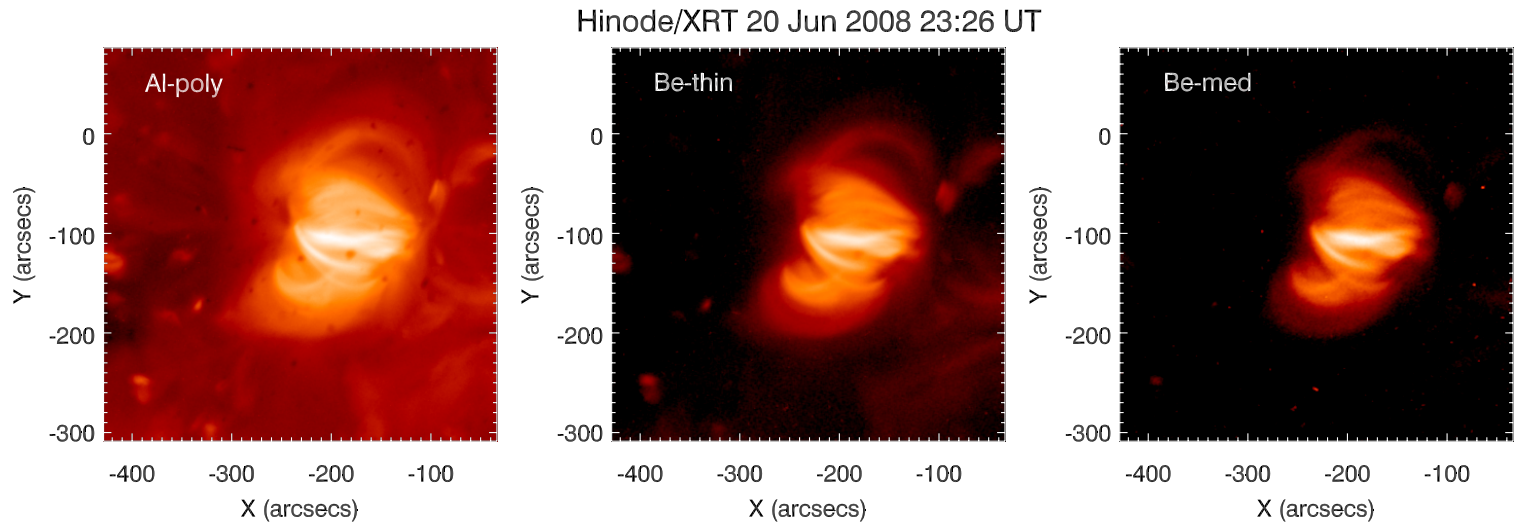

Figure 1. XRT images of AR 10999, obtained by summing all the images taken in a given filter over the $\sim 3$ hr observation (see Table 1): Al-poly (left; total integration time $t_{\text {int }} \sim 131 \mathrm{~s}$ ), Be-thin (center; $t_{\text {int }} \sim 828 \mathrm{~s}$ ), and Be-med filter (right; $t_{\text {int }} \sim 1155 \mathrm{~s}$ ).
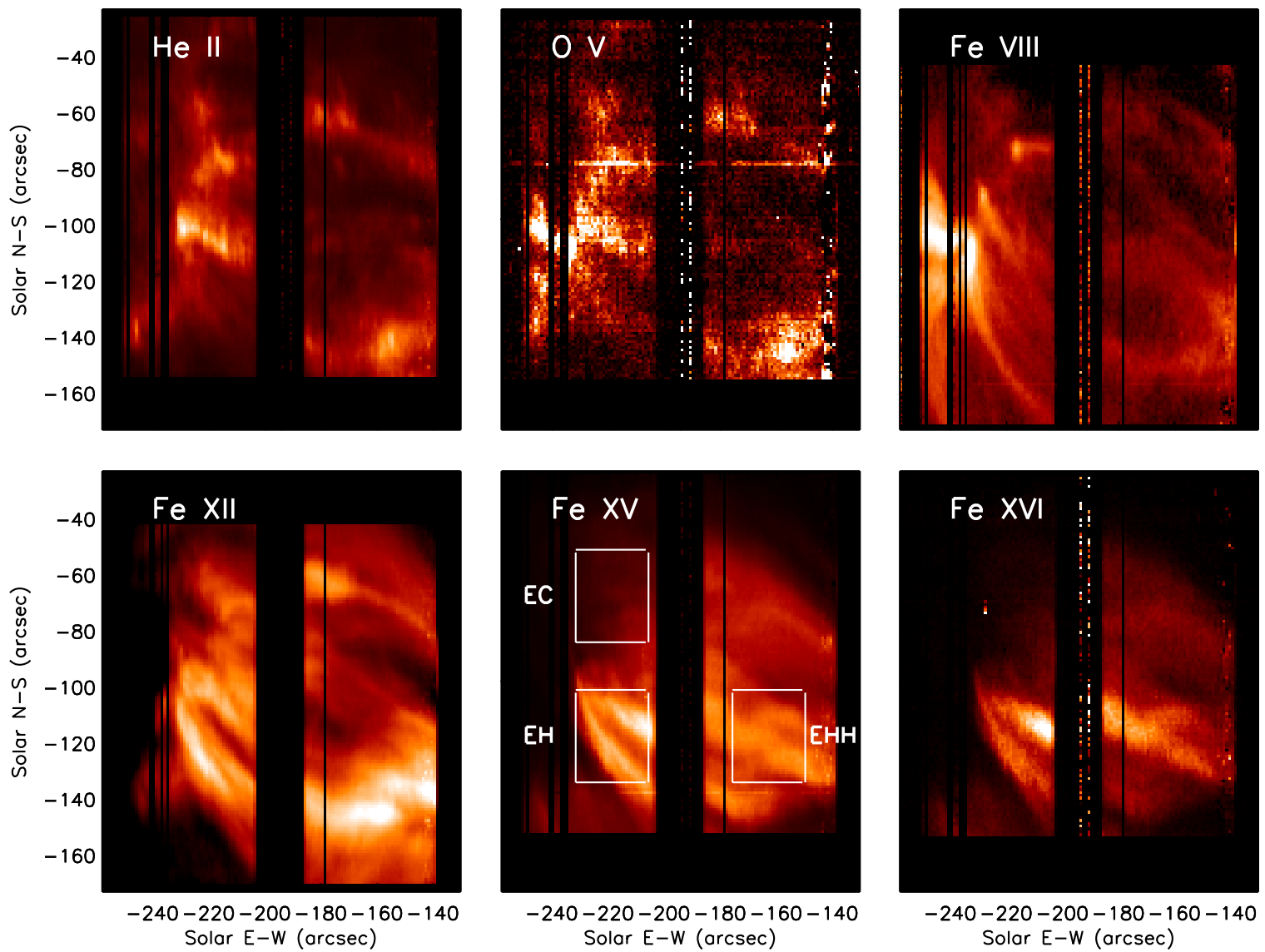

Figure 2. EIS images in spectral lines formed at different characteristic plasma temperatures: He II $\left(256.32 \AA, \log \left(T_{\max }(\mathrm{K})\right)=4.9\right), \mathrm{O} v\left(248.46 \AA, \log \left(T_{\max }(\mathrm{K})\right)=5.4\right)$, Fe VIII $\left(185.21 \AA, \log \left(T_{\max }(\mathrm{K})\right)=5.55\right)$, Fe XII $\left(195.12 \AA, \log \left(T_{\max }(\mathrm{K})\right)=6.15\right), \mathrm{Fe} \mathrm{XV}\left(284.16 \AA, \log \left(T_{\max }(\mathrm{K})\right)=6.35\right), \operatorname{and} \mathrm{Fe} \mathrm{XVI}\left(262.98 \AA, \log \left(T_{\max }(\mathrm{K})\right)=6.4\right)$. In the $284 \AA \mathrm{Fe} \mathrm{xV}$ image, we indicate the three regions selected for the analysis of the plasma thermal structure (see Figure 4 and Section 3): EC (top left), EH (bottom left), and EHH (bottom right).

over a wide range of temperatures (see Figure 3). Therefore, in order to increase signal-to-noise ratio $(\mathrm{S} / \mathrm{N})$, we have analyzed the XRT data set obtained by co-aligning the images taken in each filter at different times, and then summing them up.

In order to carry out a direct and detailed comparison of thermal analysis from XRT and EIS data, we have then selected a few subregions. To select these regions we have first obtained maps of temperature and emission measure over the whole active region, by using estimates derived with the so-called combined improved filter ratio method, devised by Reale et al. (2007). The resulting temperature map is shown in Figure 4. We selected three regions of area approximately $30^{\prime \prime} \times 30^{\prime \prime}$ : EC, which in the temperature map homogeneously appears as relatively cool, and two hotter regions (EH and $\mathrm{EHH})$. The values of temperature mean and standard deviation derived for these three regions from the CIFR diagnostics are $1.5 \pm 0.1 \mathrm{MK}(\mathrm{EC}), 1.9 \pm 0.2 \mathrm{MK}$ $(\mathrm{EH})$, and $2.0 \pm 0.2 \mathrm{MK}(\mathrm{EHH})$. When selecting the boundaries of region EHH in the XRT data, we slightly modified the shape (however maintaining the area value) in order to avoid the contamination spots clearly visible in the temperature map as bright blobs immediately to the east and north of the selected area. Figure 2 shows the selected regions in the EIS Fe Xv $284 \AA$ 

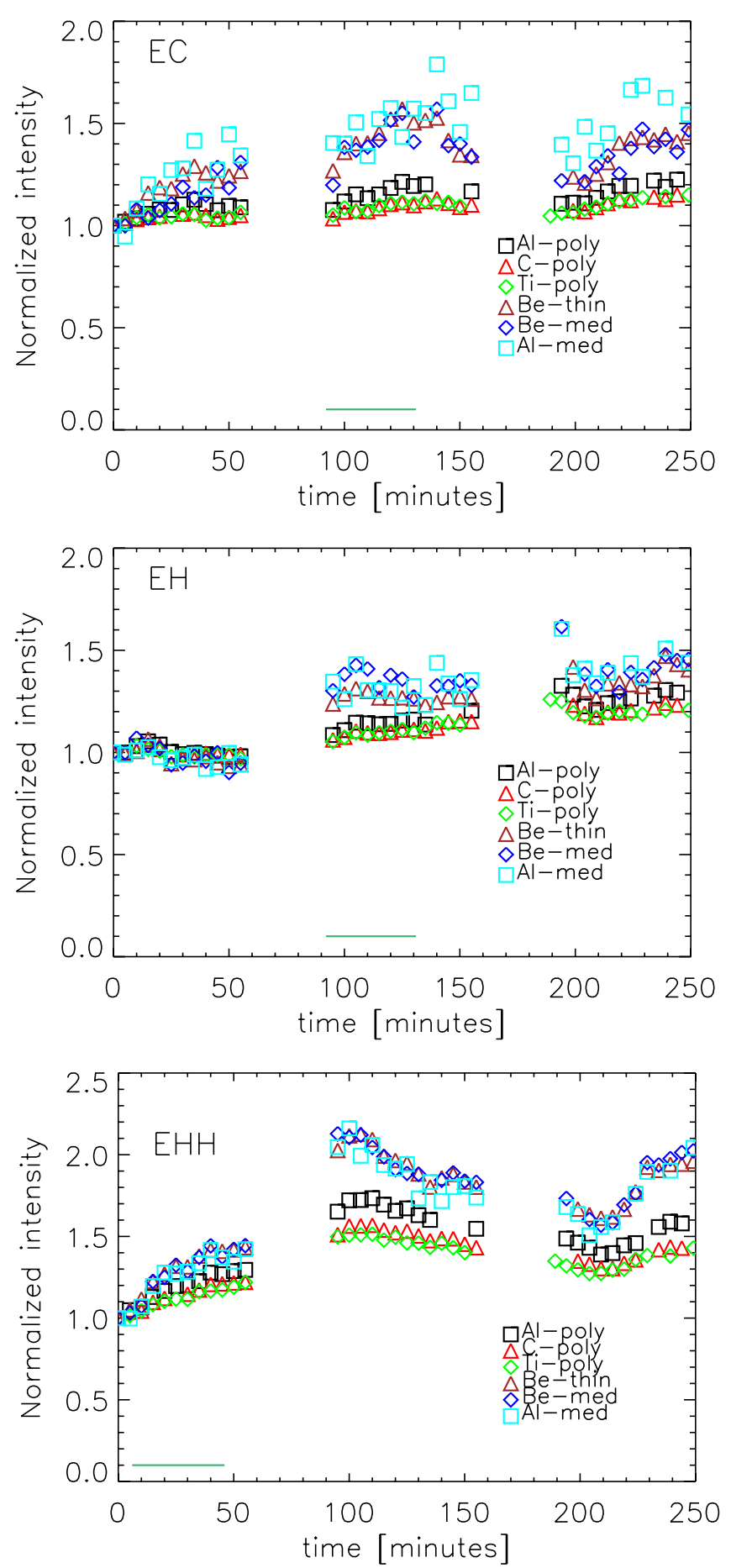

Figure 3. Light curves of XRT observations in all analyzed filters for the three selected regions: EC (top), EH (middle), and EHH (bottom). For each filter the signal is normalized to the intensity in the first image. The solid line at the bottom of the plot indicates the time interval when EIS was observing the selected region.

image. For the temperature analysis presented in the rest of the paper we integrated the XRT and EIS signals over each of these regions, over the time intervals when the two instruments were simultaneously observing the selected region. Figure 3 shows that the XRT light curves of each region changed little over the time period when EIS was observing the same areas.

Figure 5 shows the EIS full spectra in the three regions selected for the analysis with the identification of the brightest lines. The EIS exposure time (90 s at each location) yields

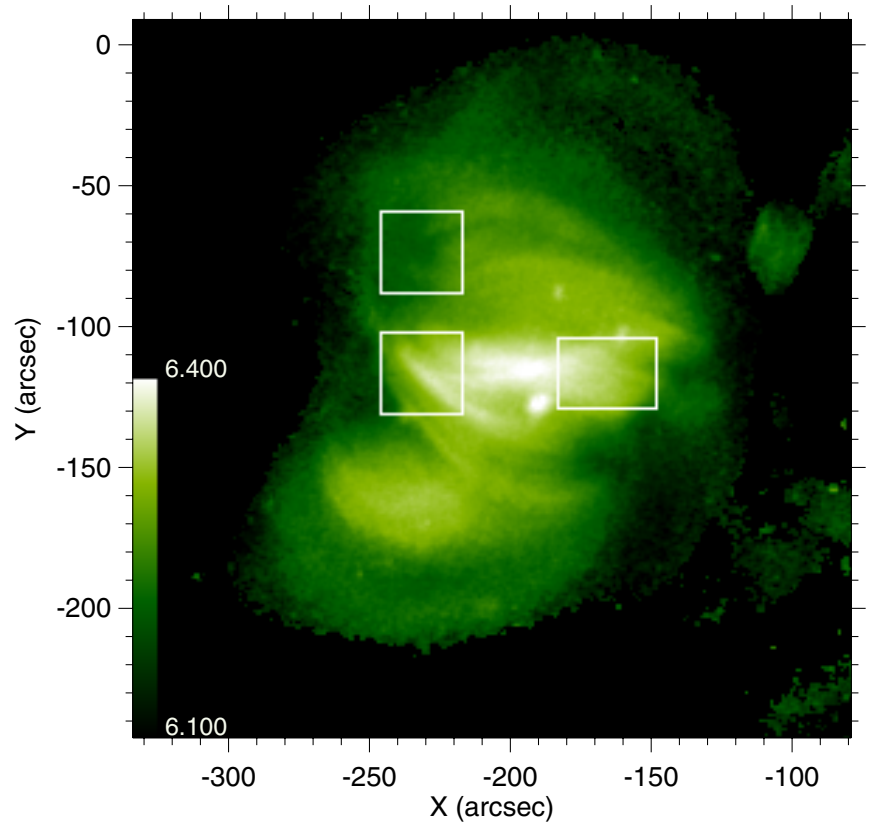

Figure 4. Temperature map obtained from the combined improved filter ratio from the XRT images of AR 10999 shown in Figure 1. The three regions selected for the detailed temperature analysis are indicated: EC (top left), EH (bottom left), and $\mathrm{EHH}$ (bottom right). When selecting the boundaries of region $\mathrm{EHH}$ we modified the shape (however maintaining the area value) in order to avoid the contamination spots clearly visible in the temperature map as bright blobs immediately to the east and north of the selected area.

high signal in the strongest lines, mostly produced in a temperature range $\log T[\mathrm{~K}] \sim 5.8-6.3$. Hotter lines present in the EIS wavelength range have generally low intensities, in typical solar non-flaring conditions, and are difficult to detect. We have selected a list of lines which are suitable for an accurate derivation of the plasma thermal properties. This list (see Table 2) includes strong lines, unblended and with reliable atomic data, and we also included hot lines for which however we can only derive upper limits from the EIS observations of this non-flaring active region. These upper limits are nevertheless useful to constrain the high temperature component $(\log T(\mathrm{~K}) \gtrsim 6.5)$.

In the following, we will describe in detail the analysis methods and results obtained for one of the selected regions, EHH. Then, we will discuss our findings for all three regions.

\subsection{Thermal Structuring from EIS Spectra}

For the analysis of EIS spectra and XRT data, we use the Package for Interactive Analysis of Line Emission (PINTofALE, Kashyap \& Drake 2000) which is available on SolarSoft. For the spectral analysis and emission measure distribution reconstruction from EIS spectra, we use CHIANTI v.6.0.1 (Dere et al. 1997, 2009) with the ionization balance of Bryans et al. (2009). Unless explicitly stated otherwise we assume "coronal" plasma abundances of Feldman (1992).

The observed line intensities provide constraints on the plasma temperature distribution, as they depend on the plasma emissivities $G_{\lambda}(T, n)$, element abundances $A_{Z}$, and differential emission measure distribution $(\operatorname{DEM}(T))$ :

$$
I_{\lambda}=A_{Z} \int_{T} G_{\lambda}\left(T, n_{e}\right) \operatorname{DEM}(T), d T,
$$

where $\operatorname{DEM}(T)=n_{e}^{2} d V / d T\left(\mathrm{~cm}^{-3} \mathrm{~K}^{-1}\right)$. 


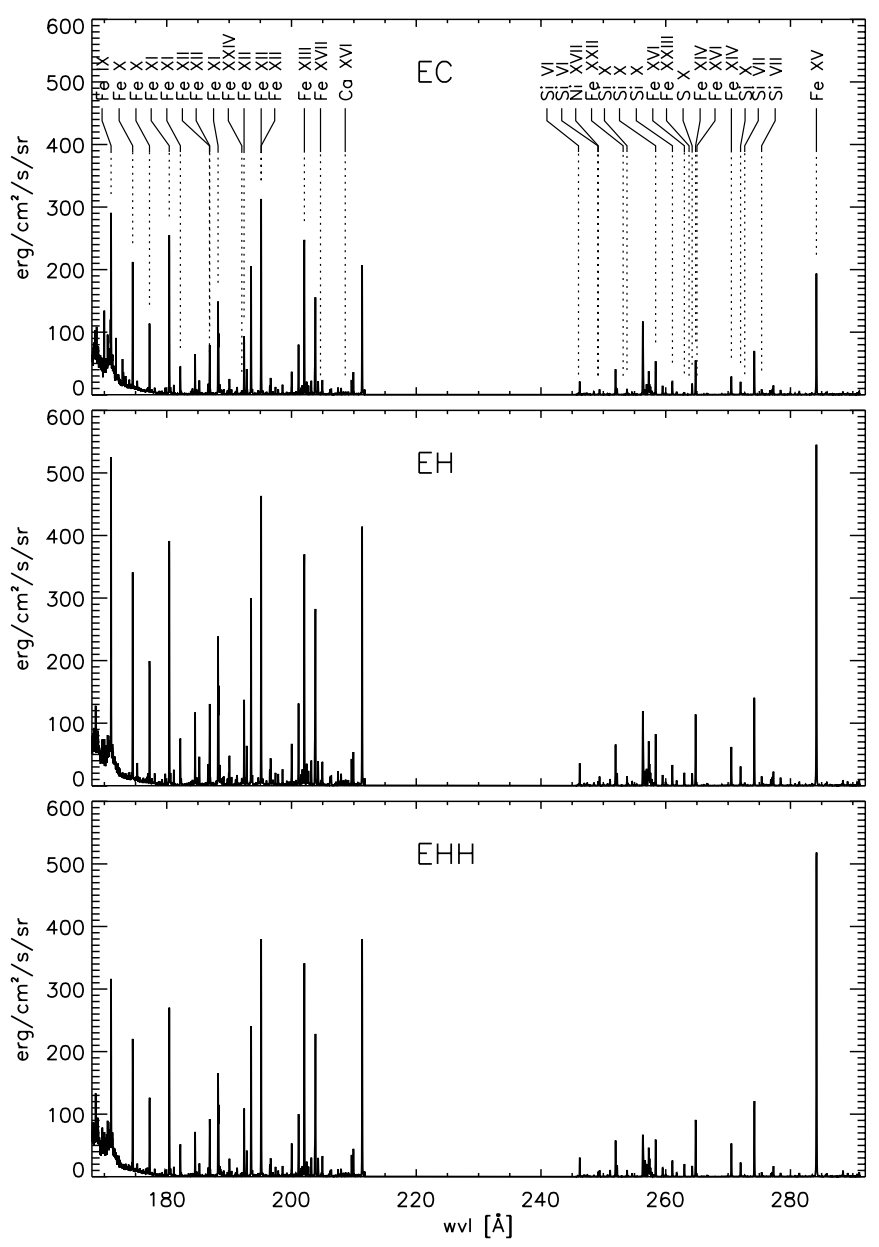

Figure 5. EIS spectra of the three regions selected for the analysis: EC (top), $\mathrm{EH}$ (middle), and EHH (bottom). The identification of the spectral lines used for the analysis of the plasma thermal properties (listed in Table 2) are shown in the spectrum in the top panel.

Several lines listed in Table 2 are density sensitive, so it is necessary to measure the electron density of the emitting plasma for each region before calculating the contribution functions to be used in the analysis. We have used the line intensity ratios of Si X, Fe XII, and Fe XIV available in the data set, and the results are listed in Table 3. The most reliable indicator of the electron density is Si X, since its lines are not affected by blends and have been found to be reliable by Young et al. (1998) and Landi et al. (2002). The Fe XIV intensity ratio is less sensitive to the electron density and provides a much coarser estimate; the density values it indicates are broadly consistent with the Si X measurements. The Fe XII densities, while consistent within the uncertainties, are a bit larger than those of Si x, a behavior that has already been noted elsewhere (Young et al. 2009; Watanabe et al. 2009); this might be due to atomic physics problems affecting the Fe XII emissivities (however, see also Tripathi et al. 2010). Throughout the present work, we used $\log n_{e}=9.0$ for all regions in the calculation of the spectral emissivities from CHIANTI.

Figure 6 shows the EM loci corresponding to the fluxes measured for one of the regions. The curves represent the loci of constant flux for a given line, i.e., they are obtained by taking the ratio of the measured line flux to the emissivity function (including the element abundance) of that line. The EM loci plot provides a visual indication of the temperature range covered by the selected lines and passbands, since we are also including EM loci for the XRT observations. For the XRT channels, the
Table 2

Identification and Measured Fluxes of EIS Spectral Lines Used for Reconstructing the DEM of the Three Regions Selected for the Analysis and Shown in Figure 2

\begin{tabular}{|c|c|c|c|c|c|c|}
\hline \multirow[t]{2}{*}{$\lambda(\AA)$} & \multirow[t]{2}{*}{ Ion } & \multirow[t]{2}{*}{$\log \left(T_{\max }(\mathrm{K})\right)$} & \multicolumn{3}{|c|}{ Flux $\left(\mathrm{erg} \mathrm{cm}^{-2} \mathrm{~s}^{-1}\right)$} & \multirow[t]{2}{*}{ Notes $^{\mathrm{a}}$} \\
\hline & & & EC & $\mathrm{EH}$ & $\mathrm{EHH}$ & \\
\hline 171.0755 & Fe XIX & 5.9 & 8494.52 & 16757.8 & 9238.51 & \\
\hline 174.5340 & Fe $x$ & 6.0 & 7310.57 & 12152.6 & 7888.11 & \\
\hline 177.2430 & Fe $x$ & 6.0 & 4231.15 & 7555.12 & 4736.85 & \\
\hline 180.4080 & $\mathrm{Fe} X I$ & 6.1 & 10609.4 & 16354.4 & 11523.8 & \\
\hline 182.1690 & $\mathrm{Fe} \mathrm{XI}$ & 6.1 & 1784.80 & 3021.49 & 1914.51 & \\
\hline 186.8520 & Fe XII & 6.2 & 694.141 & 1099.68 & 426.001 & \\
\hline 186.8840 & Fe XII & 6.2 & 3129.81 & 5212.59 & 3902.01 & \\
\hline 188.2320 & $\mathrm{Fe} X I$ & 6.1 & 5613.69 & 8863.01 & 6469.60 & \\
\hline 192.0285 & Fe XXIV & 7.3 & 310.929 & 539.337 & 304.685 & $\mathrm{u}$ \\
\hline 192.3930 & Fe XII & 6.2 & 4107.59 & 5858.81 & 4550.20 & \\
\hline 195.1180 & Fe XII & 6.2 & 14092.9 & 21301.2 & 16415.0 & \\
\hline 202.0440 & Fe XIII & 6.2 & 11240.2 & 15845.5 & 14868.5 & \\
\hline 204.6542 & Fe XVII & 6.7 & 37.2628 & 60.1331 & 44.5043 & $\mathrm{u}$ \\
\hline 208.6040 & Ca XVI & 6.7 & 5.61904 & 17.4814 & 38.7130 & $\mathrm{u}$ \\
\hline 246.0200 & Si VI & 5.6 & 76.5847 & 91.3415 & 28.2609 & \\
\hline 249.1240 & Si VI & 5.6 & 29.2495 & 38.8003 & 15.6522 & \\
\hline 249.1780 & Ni XVII & 6.5 & 20.5272 & 277.752 & 312.949 & \\
\hline 253.1702 & Fe XXII & 7.1 & 3.55755 & 9.38507 & 9.47460 & $\mathrm{u}$ \\
\hline 253.7880 & Si X & 6.1 & 379.199 & 578.696 & 436.514 & \\
\hline 258.3710 & Si X & 6.1 & 2737.30 & 4175.65 & 3144.01 & \\
\hline 261.0440 & Si $x$ & 6.1 & 1151.82 & 1731.62 & 1318.26 & \\
\hline 262.9760 & Fe XVI & 6.4 & 173.934 & 968.696 & 1036.80 & \\
\hline 263.7657 & Fe XXIII & 7.2 & 14.5618 & 12.5289 & 9.42194 & $\mathrm{u}$ \\
\hline 264.2310 & S x & 6.2 & 925.718 & 975.778 & 899.526 & \\
\hline 264.7900 & Fe XIV & 6.3 & 2784.31 & 5570.13 & 4768.17 & \\
\hline 265.0010 & Fe XVI & 6.4 & 19.6691 & 105.484 & 99.7671 & \\
\hline 270.5220 & Fe XIV & 6.3 & 1356.52 & 3060.05 & 2607.03 & \\
\hline 272.0060 & Si $x$ & 6.1 & 1117.27 & 1592.69 & 1191.82 & \\
\hline 272.6390 & Si VII & 5.8 & 134.686 & 237.159 & 99.4883 & \\
\hline 275.3540 & Si VII & 5.8 & 456.285 & 706.011 & 317.491 & \\
\hline 284.1630 & $\mathrm{Fe} x \mathrm{~V}$ & 6.3 & 10317.8 & 28644.9 & 27965.3 & \\
\hline
\end{tabular}

Note. " $u$ " indicates upper limits of fluxes of hot lines which are not actually detected in the EIS spectra.

Table 3

Electron Density Diagnostic Results for Each Region

\begin{tabular}{lcccc}
\hline \hline Ion & Line Ratio & \multicolumn{3}{c}{$\log n_{e}$} \\
\cline { 3 - 5 } & & EC & EH & EHH \\
\hline Si X & $261.0 / 253.8$ & $8.95_{-0.20}^{+0.30}$ & $9.00 \pm 0.25$ & $8.95_{-0.20}^{+0.30}$ \\
Fe XII & $186.8 / 195.1$ & $9.15_{-0.20}^{+0.15}$ & $9.20 \pm 0.20$ & $9.10 \pm 0.15$ \\
Fe XIV & $264.8 / 270.5$ & $9.5 \pm 0.4$ & $9.3_{-0.5}^{+0.3}$ & $9.3_{-0.5}^{+0.3}$ \\
\hline
\end{tabular}

Notes. The density adopted throughout the study is $\log n_{e}=9.0$. Densities are in $\mathrm{cm}^{-3}$.

EM loci are derived by dividing the measured fluxes by the temperature response of that channel, derived assuming a given set of element abundances (in the case of Figure 6 we use coronal abundances). Also, if the underlying plasma were isothermal the EM loci curves would all cross at a single points, therefore this plot also provides some indication for the characteristics of temperature distribution of the plasma.

The method of reconstruction of the differential emission measure distribution that we adopt for the analysis of EIS data runs a Markov-chain Monte Carlo (MCMC) algorithm on a set of line fluxes (intensities in broad bandpasses can also be used, as for instance in the case of XRT data sets; see Section 3.2), and 


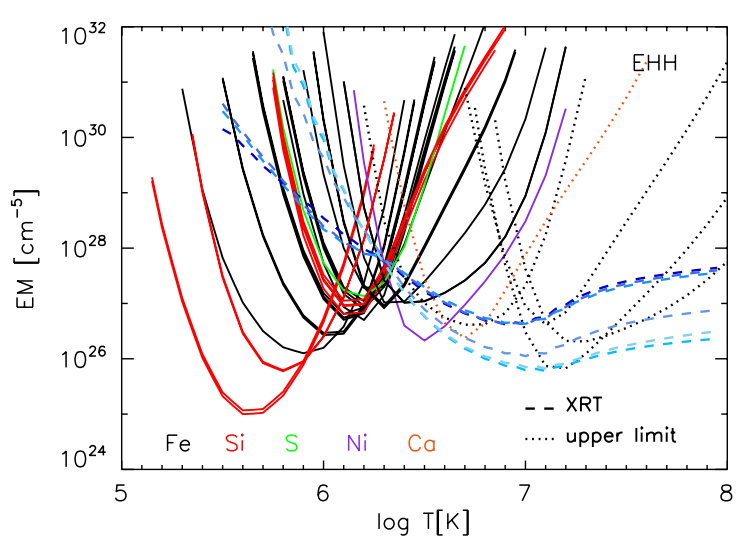

Figure 6. EM loci obtained for the measured EIS line fluxes, including upper limits (dotted lines) for hot lines in EIS passbands. The dashed lines indicate the EM loci for XRT measured fluxes. We use different colors for lines of different elements, as indicated in the inset.

it returns an estimate of the DEM that generates the observed fluxes (see Kashyap \& Drake (1998) for details on assumptions and approximations). With respect to other methods for reconstructing the plasma emission measure distribution from a set of fluxes, the MCMC method we adopt has the main advantage of providing an estimate of the uncertainties in the derived DEM. The problem of determining the emission measure distribution and its confidence limits is notoriously challenging (e.g., Craig \& Brown 1976; Judge et al. 1997; Judge 2010). Part of the reason is that the emission measure at a given temperature cannot be determined independently of temperatures at other bins, and therefore the corresponding errors are also correlated. The MCMC method works around this fundamental problem by sampling solutions from the full probability distribution of the DEM given the data. It is a feature of the MCMC chain that regardless of the conceptual complexity of the solution space, it can be fully explored numerically at a relatively low computational cost. Thus, the sampled solutions include the effects of statistical noise from the measured data as well as correlations across temperatures that arise due to overlaps between the individual contribution functions of the different lines. From the set of solutions thus obtained, we can depict the uncertainty range at temperatures of interest. Since it is difficult to display correlations, for purposes of clarity, we only show the error ranges computed separately at each temperature bin in the figures. Error bars computed for predicted fluxes, and thus abundances, are uncorrelated and thus have the usual meaning. Another problem with DEM reconstruction is that the derived curves are solutions to a Fredholm integral equation of the first kind, and are thus subject to high-frequency instability. These instabilities are typically suppressed by imposing a global smoothness criterion. In the case of the MCMC method adopted here, however, this restriction is relaxed. Smoothing is more physically based, is locally variable, and is limited by the width and number of the line contribution functions used. This leads to solutions that individually have more fluctuations, but on average the ensemble of solutions produce an envelope that reliably determines features that are statistically significant.

As uncertainties on the measured line fluxes listed in Table 2 we combine in quadrature the statistical uncertainties which are typically very small (of the order of few percent), with the uncertainty in the absolute radiometric calibration for EIS which is estimated to be $22 \%$ (Lang et al. 2006).

Figure 7 shows the results of the MCMC method to derive the emission measure distribution applied to the EIS fluxes mea-
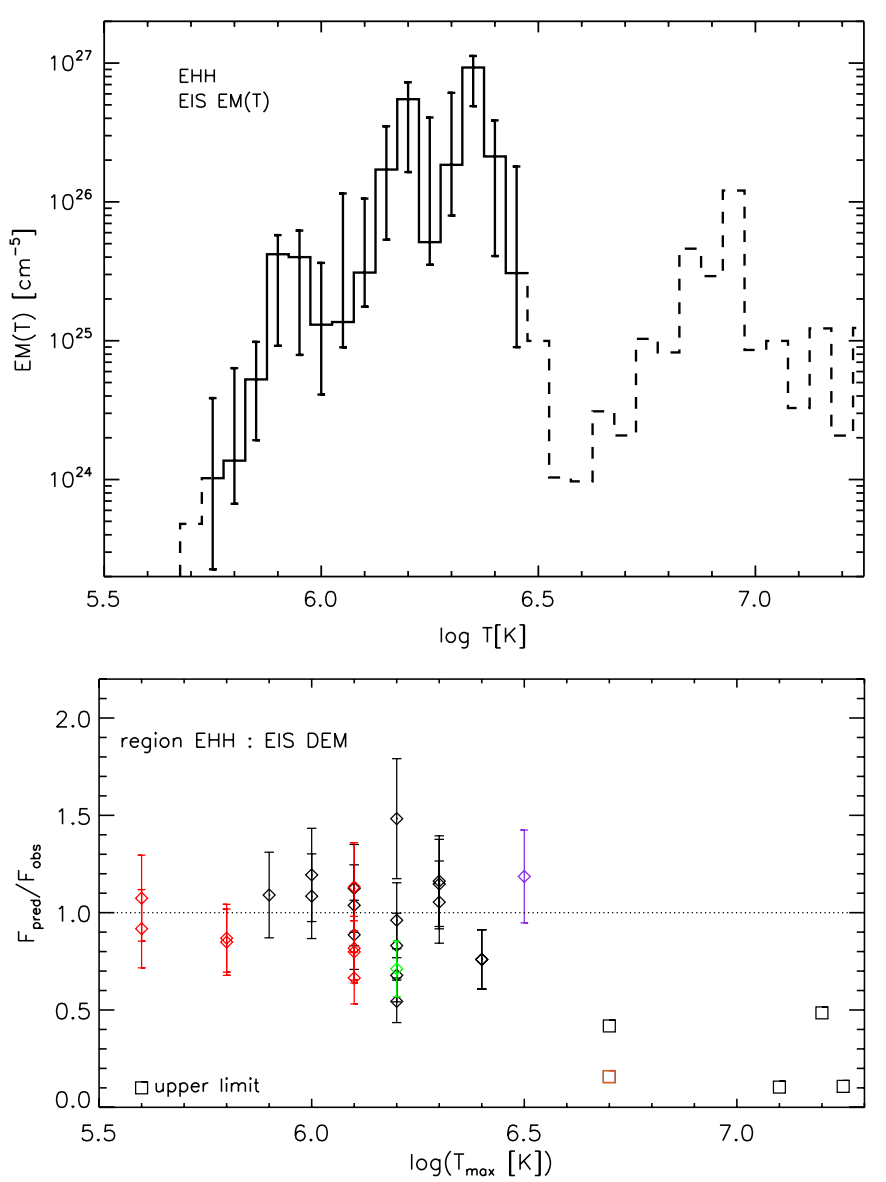

Figure 7. Top: emission measure distribution derived for region EHH with the MCMC method (see the text) using EIS line fluxes listed in Table 2. The error bars provide a measure of the extent to which the $(\operatorname{EM}(T))$ of each temperature bin is constrained by the measured fluxes. We plot uncertainties only for the temperature bins for which the $(\operatorname{EM}(T))$ contributes to at least $5 \%$ of the flux of at least one line (see Figure 8 and the text for details). Unconstrained bins are plotted with dashed line. Bottom: comparison of measured EIS line fluxes with flux values predicted using the emission measure distribution derived with the MCMC method. Different colors are used for lines of different elements in accordance with the colors used to show the EM loci (Figure 6).

sured for the EHH region. In this case, as in the rest of the paper, we will plot the emission measure distribution $(\operatorname{EM}(T))$ which is obtained by integrating the differential emission measure distribution $\operatorname{DEM}(T)$ in each temperature bin (here we use a temperature grid with constant $\Delta \log T=0.05$ ). The upper panel shows the emission measure distribution that reproduces the measured fluxes; the associated uncertainties estimated as described at the beginning of this section are also plotted. The bottom panel shows the ratios of predicted to observed fluxes for the EIS lines. The comparison of measured fluxes with predictions based on the emission measure distribution shows that all fluxes are reproduced within about $50 \%$, and that the fluxes predicted for the hot lines, for which the spectra only provide upper limits, are all lower than the upper limits.

We can investigate the compatibility of this derived $(\operatorname{EM}(T))$ with the XRT observations by computing the predicted XRT fluxes and comparing them with the measured ones. It is worth noting however that when deriving the $(\operatorname{EM}(T))$ using exclusively the information contained in the EIS spectra, not all temperature bins are well constrained. In particular for these EIS data sets which lack measured lines in the high temperature range $(\log T[\mathrm{~K}] \gtrsim 6.5)$ the hot tail of the $(\mathrm{EM}(T))$ is poorly constrained, but XRT is very sensitive to it, having temperature 


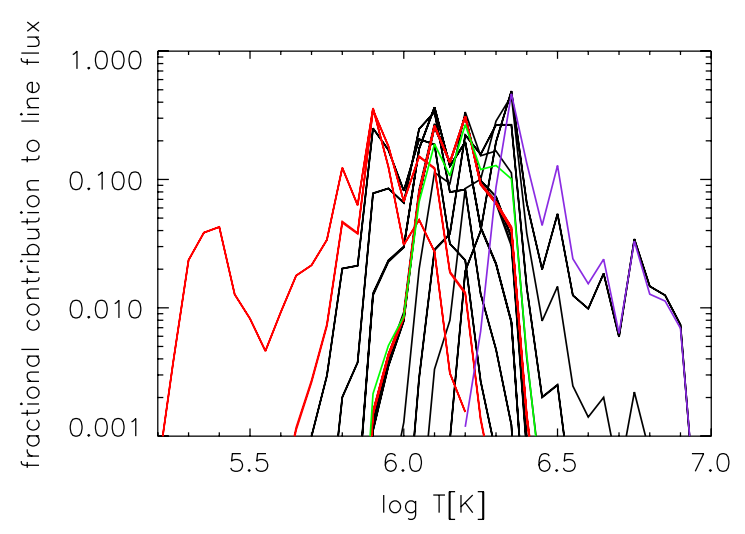

Figure 8. Each curve represents the fractional contribution of each temperature bin to the flux of one of the EIS lines used for DEM reconstruction (hot lines for which we only derived upper limits are not shown). We use the same colors as in Figure 6.

responses which peak around $\log T[\mathrm{~K}] \sim 7$ for all filters. Therefore, some caution must be applied to perform this crosscheck. To determine in which temperature bins the $(\operatorname{EM}(T))$ is constrained by EIS data we look at the contribution of each bin to the line fluxes. Folding the $(\operatorname{EM}(T))$ with the line emissivity we can investigate the relative weight of each temperature bin for each line, as shown in Figure 8. We then consider as constrained the temperature bins where the $(\operatorname{EM}(T))$ contributes more than a threshold (percentage) value to at least one of the EIS lines. The choice of the threshold value is somewhat arbitrary and we chose a rather conservative $5 \%$.

Considering the $(\operatorname{EM}(T))$ in the temperature bins satisfying our selection criterion, we calculate the predicted fluxes in the XRT filters (which therefore are strictly speaking lower limits to the values predicted by the assumed $(\operatorname{EM}(T)))$ which are listed in Table 4, together with the measured fluxes. The comparison of the two sets indicates that the $(\operatorname{EM}(T))$ derived from EIS underpredicts the XRT fluxes by a factor $\sim 2$, the discrepancy being slightly larger for the thinner filters (Al-poly, C-poly, Ti-poly) which are more sensitive to the cooler plasma which is better characterized by the EIS data.

\subsection{Thermal Structuring from XRT Data}

We then derive the thermal distribution of the coronal plasma exclusively from XRT data by using two different methods:

1. Forward fitting of distributions of filter ratios of several filter pairs, through pixel-by-pixel Monte Carlo simulations of the observations, using two temperature components (2T);

2. MCMC method, as for the analysis of the EIS spectra (see Section 3.1).

We use XRT temperature responses calculated assuming “coronal" abundances (Feldman 1992) and the new XRT calibration which includes a model for the contamination layers deposited both on the CCD and on the focal plane filters (Narukage et al. 2010). For the detailed comparison of XRT and EIS data for each of the selected regions we only use the subset of XRT data taken simultaneously to the EIS observations (see Figure 3).

We have applied the forward fitting method with two $T$ components already in previous analysis of XRT data aimed at deriving the plasma temperature distribution of an active region, in particular to investigate the presence of a high temperature component (Reale et al. 2009b). The details of this method are thoroughly discussed by Reale et al. (2009b). In summary,
Table 4

For Region EHH, Comparison of XRT Measured Fluxes with the Values Predicted Using the $(\operatorname{EM}(T))$ Derived from EIS Line Fluxes (Shown in the Upper Panel of Figure 7) and from the Forward Modeling with Two Temperature Components (see Section 3.2), Assuming Coronal Abundances

\begin{tabular}{lccc}
\hline \hline XRT Filter & $\begin{array}{c}F_{\text {obs }} \\
(\mathrm{DN} / \mathrm{s})\end{array}$ & $\begin{array}{c}F_{\text {pred,EIs }}{ }^{\mathrm{a}} \\
(\mathrm{DN} / \mathrm{s})\end{array}$ & $\begin{array}{c}F_{\text {pred,fm }}{ }^{\mathrm{b}} \\
(\mathrm{DN} / \mathrm{s})\end{array}$ \\
\hline Al-poly & 122.0 & $57.8(41.8-70.2)$ & 123.2 \\
C-poly & 95.8 & $44.5(32.8-53.4)$ & 101.1 \\
Ti-poly & 69.1 & $31.0(23.0-36.9)$ & 65.94 \\
Be-thin & 11.1 & $5.85(3.69-7.61)$ & 9.82 \\
Be-med & 1.59 & $1.06(0.66-1.38)$ & 1.42 \\
Al-med & 0.730 & $0.483(0.301-0.628)$ & 0.673 \\
\hline
\end{tabular}

Notes.

a XRT fluxes predicted using the emission measure distribution derived using EIS line fluxes only. The values in parentheses represent the range of fluxes predicted by the Monte Carlo simulations of the $\operatorname{EM}(T)$ which are the acceptable emission measure distributions, defining the error bars shown in Figure 7.

b XRT fluxes predicted using the $(\operatorname{EM}(T))$ derived from the forward modeling with $2 T$, and using coronal abundances.

the pixel fluxes in the relevant XRT filters are computed from a basic model $\operatorname{EM}(T)$ along the line of sight defined by few parameters (amplitude, central temperature, and width of either single or double top-hat functions). Monte Carlo simulations are used to randomize some of the $\operatorname{EM}(T)$ parameters and to include Poisson photon noise. This procedure is replicated to build whole fake XRT images in all filters. The simulated XRT data are then analyzed exactly as they were actual data: (1) temperature and emission measure maps, i.e., single values for each pixel, are obtained for a given filter ratio, and this is done for several filter pairs which are sampling the thermal structure in different ways; (2) for each considered filter ratio an emission measure distribution versus temperature is built by summing up the emission measure values of all pixels falling in the same temperature bin. The input $(\operatorname{EM}(T))$ is deemed an adequate representation of the underlying plasma emission measure distribution when these curves of emission measure distributions versus temperature are reproducing satisfactorily the analogous curves obtained from actual data.

The results of these simulations for region $\mathrm{EHH}$ are shown in Figure 9 and Table 4. We note that, as discussed thoroughly in Reale et al. (2009b) for the analysis of XRT observations of another active region, while this analysis method is certainly approximate it allows us to be sensitive to possible minor $\operatorname{EM}(T)$ components, e.g., small hot components, and to somewhat constrain them. As for the case presented in Reale et al. (2009b), here also we find that a high temperature plasma component, even if much weaker than the dominating cool $(\log T(\mathrm{~K}) \sim 6.1-6.3)$, is nevertheless appropriate to reproduce the XRT observations.

As mentioned above, in order to derive the $(\operatorname{EM}(T))$ from XRT data we also use the MCMC method, using the flux values integrated in each passband over the whole subregion. The errors associated to the XRT fluxes are estimated by taking into account the photon noise and the calibration uncertainties. Like for the EIS fluxes, the statistical uncertainties (we observe bright sources and integrate over time to further increase the $\mathrm{S} / \mathrm{N}$ ) are typically very small and the errors are largely dominated by the calibration uncertainties (for an estimate of the latter see Narukage et al. 2010). The resulting errors are of the order of $\sim 5 \%$ for the thin filters, and $\sim 15-20 \%$ for thin-Be and the medium filters. The derived best fit $(\operatorname{EM}(T))$ is shown in 

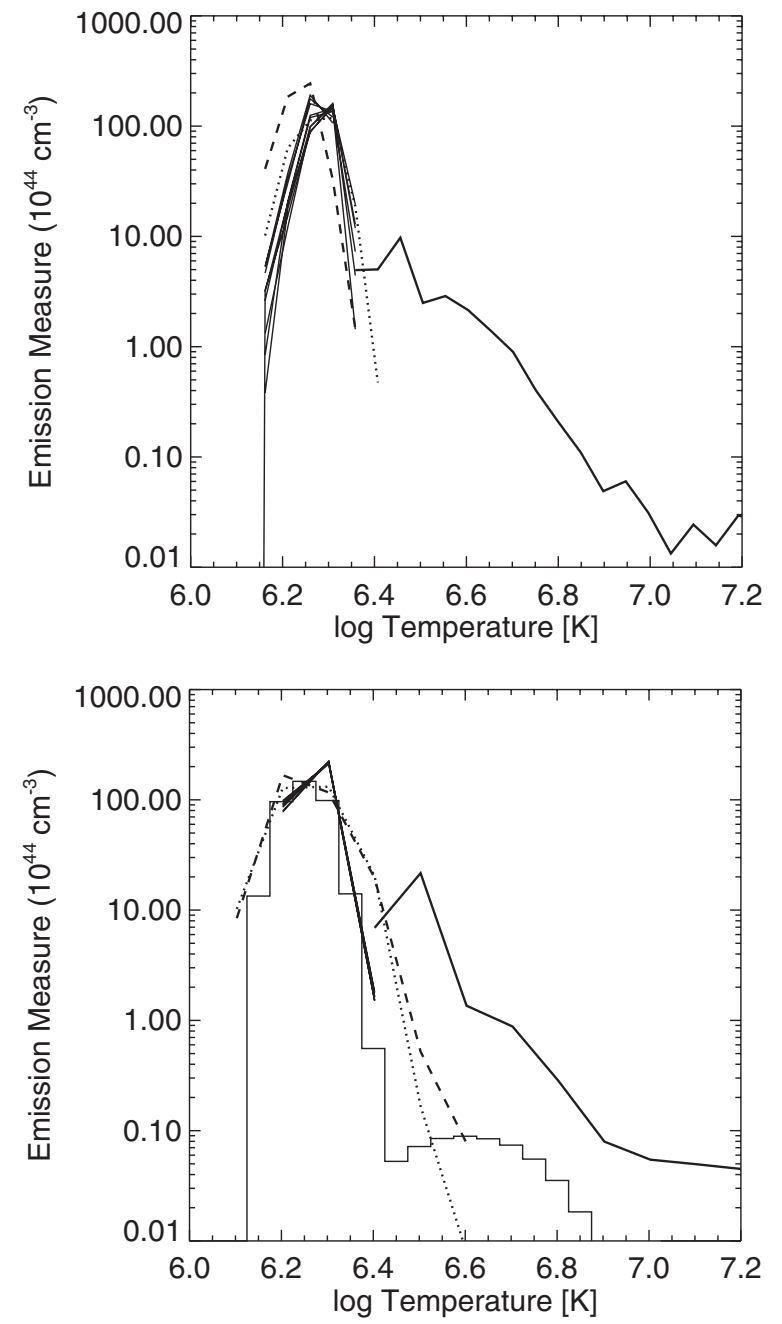

Figure 9. Top: emission measure distribution vs. temperature derived for region EHH from XRT data. Each curve corresponds to a filter pair: soft filter ratios (thin solid lines), Al_med/Be_thin (dotted line), Be_med/Be_thin (dashed line), and Be_med/Al_med (thick solid line). Bottom: emission measure distribution vs. temperature derived for region EHH from XRT data simulated using the $(\operatorname{EM}(T))$ histogram as parent emission measure distribution along the line of sight. Lines as in the top panel.

Figure 10 (red line); the associated uncertainties are rather large, as should also be expected considering the limited constraints that the XRT fluxes in six filters can provide to the emission measure distribution over a large temperature range.

The differences between the results of the two methods are not large compared to the associated uncertainties. The agreement is overall acceptable if one considers that the approaches are completely different. The MCMC method is model independent but it only uses the total region flux values. The forward fitting $2 \mathrm{~T}$ method makes an assumption on the basic $\operatorname{EM}(T)$ distribution but it keeps the spatial information of the pixel values longer in the analysis pipeline, and it is therefore more sensitive to small local plasma inhomogeneities (at the scale of the single pixel). Moreover this latter method gives large weight to the Be-med/Al-med filter ratio, and this allows to detect the small hot component.

In Figure 10, we compare the emission measure distributions derived independently from XRT and EIS data. Considering only the temperature regions where the $\operatorname{EM}(T)$ are constrained by the data, i.e., the bins where the error bars are shown, the

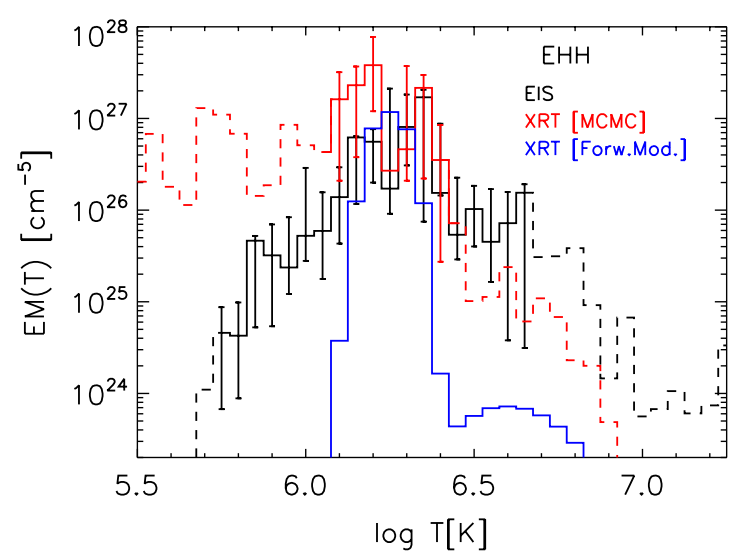

Figure 10. Comparison of emission measure distribution derived for region EHH independently from EIS lines (with MCMC method, see Section 3.1; black line), and from XRT, with two different methods: MCMC (red line) and forward modeling with two temperature components (blue line). For the $(\operatorname{EM}(T))$ derived using the MCMC method we show the estimate of uncertainties for those temperature bins which contribute at least $5 \%$ to the flux of at least one line or passband (see Figure 8).

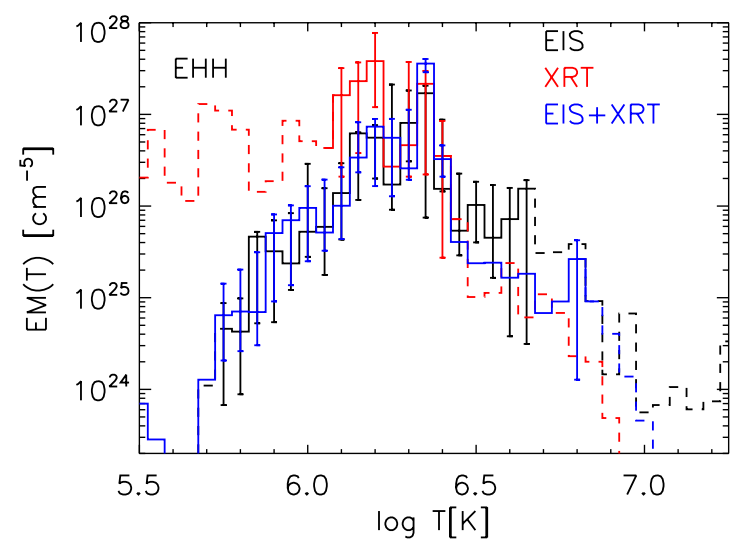

Figure 11. Comparison of emission measure distribution derived for region $\mathrm{EHH}$ with different methods: from EIS lines (black line; same curve as Figure 10), from XRT (red line; same curve as curve shown in Figure 10), and using EIS and XRT data together (blue curve).

different data sets yield emission measure distributions that are not too dissimilar when the (large) uncertainties are taken into account. In particular, they have similar peak temperature and width of the cool component. Using the $(\operatorname{EM}(T))$ derived from XRT data with the MCMC method to calculate the expected fluxes for the EIS lines of Table 2, we find that the XRT $(\operatorname{EM}(T))$ reproduces the measured EIS fluxes not too accurately. Specifically, the lines with typical temperature of formation $\log T[\mathrm{~K}] \gtrsim 6.3$, such as Fe XIV and Fe XV, and the lines formed around $\log T[\mathrm{~K}] \sim 6.1-6.2$, such as Fe XII and Si X, are overpredicted by a factor $\sim 3$ (typical range spans approximately from a factor $2-5)$; cooler $\operatorname{lines}(\log T[\mathrm{~K}] \lesssim 6.0)$ are instead slightly underpredicted (typical range: $\sim 0.05$ to $\sim 1.5 \times$ the measured fluxes).

\subsection{Thermal Structuring from EIS and XRT}

We finally take advantage of all the available information, and derive the plasma $(\operatorname{EM}(T))$ by applying the MCMC method to the measured XRT and EIS fluxes together. The $(\operatorname{EM}(T))$ derived for region $\mathrm{EHH}$ by combining the two instruments is shown in Figure 11, compared to the emission measure distributions derived independently from each of the two data sets. When combining XRT and EIS data, the resulting $(\operatorname{EM}(T))$ 


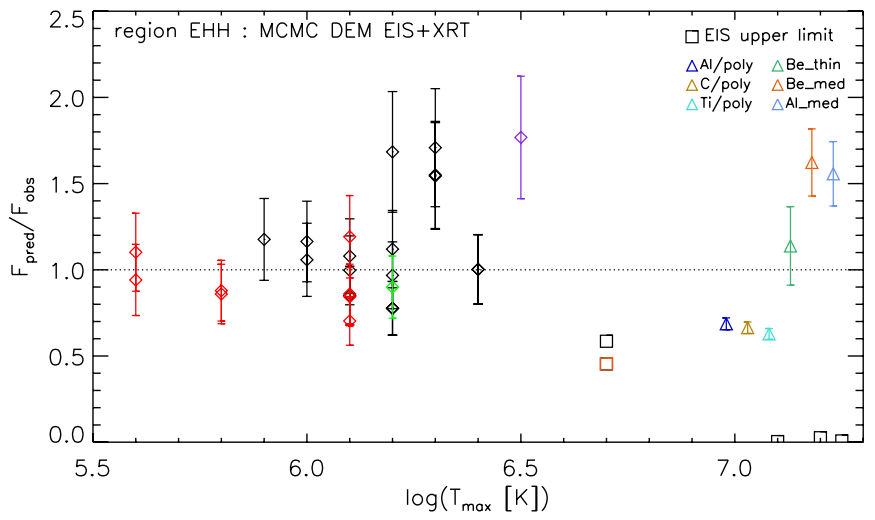

Figure 12. Comparison of measured EIS and XRT fluxes with flux values predicted using the emission measure distribution derived with the MCMC method using both set of fluxes and shown in Figure 11. We use the same colors as in Figure 6 to indicate lines of different elements or XRT passbands.

is overall rather similar to the $(\operatorname{EM}(T))$ derived from EIS data, which provide much tighter constraints because of the large number of lines and temperature dependence of their emissivity. In the high temperature range, however, where XRT is more sensitive to the plasma emission and EIS provides poor constraints, the XRT+EIS $(\operatorname{EM}(T))$ departs more from the EIS curve.

Figure 12 shows how well the XRT+EIS $(\operatorname{EM}(T))$ reproduces the measured fluxes. While the EIS line fluxes are reproduced reasonably well, similarly to the case of the $\operatorname{EIS~}(\operatorname{EM}(T))$, there is some systematic discrepancy between the predicted and the observed fluxes in the XRT passbands. Specifically, the three cooler filters, Al-poly, C-poly, and Ti-poly, are underpredicted by about a factor two. The fluxes in the hotter filters are instead well reproduced. We note that for these latter filters, which are much less sensitive to cool plasma with respect to the thin filters, the algorithm of derivation of the emission measure distribution can find a satisfactory agreement with the observations because of the leeway in the determination of the high temperature component which is not tightly constrained by the other fluxes, and in particular does not contribute significantly to the analyzed EIS lines. These discrepancies are reminiscent of the systematics found when predicting XRT fluxes using the EIS $(\operatorname{EM}(T)$ ) (see Table 4), and when using the XRT $(\operatorname{EM}(T))$ to predict the EIS line fluxes (see discussion at end of Section 3.2). The crosscheck provided by the three methods points to a systematic difference with EIS data yielding emission measure values a factor $\sim 2$ lower than the values derived from XRT data, as also evident from Figure 11.

We explored how some of the assumptions we have made for our analysis may affect these results. Among those, the assumption for the element abundances of the coronal plasma has a potentially very significant effect on the derivation of the plasma emission measure distribution. There is a large body of evidence that element abundances in the solar corona do not reflect the solar photospheric composition (e.g., Meyer 1985; Feldman 1992). In particular, the chemical fractionation appears to be a function of the first ionization potential (FIP), with lowFIP elements, such as Fe and $\mathrm{Si}$, typically enhanced in the corona by a factor of a few, whereas high-FIP elements such as $\mathrm{O}$ are thought to have coronal abundances close to their photospheric values (e.g., Meyer 1985; Feldman 1992).

The intensity of spectral lines is linearly dependent on the element abundance (assuming that the abundance is the

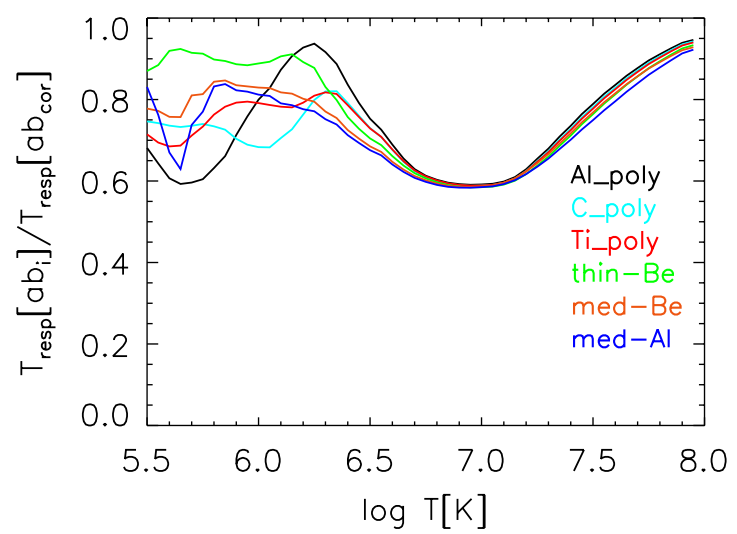

Figure 13. Ratio of XRT responses, as a function of temperature, calculated using intermediate abundances (FIP bias $=2$ ), to the responses calculated using coronal abundances (Feldman 1992). A different color is used for each filter used in this work, as indicated in the inset.

same at all temperatures for plasma in the studied region; see Equation (1)). Therefore, if the $(\operatorname{EM}(T))$ is derived from lines all emitted from a single element, e.g., Fe, a change in the element abundance will not change the shape of the emission measure distribution but merely shift its absolute value: for instance, a decrease of the abundance by a factor two will determine an increase of the $(\operatorname{EM}(T))$ by a factor two. Although our analysis of EIS data includes lines emitted by different elements, the large majority of the considered lines are emitted by low-FIP elements $\mathrm{Fe}$ and $\mathrm{Si}$. Therefore, if we consider a chemical composition with a FIP bias different from the Feldman (1992) set, typically adopted for coronal plasma and used for all the above analysis, we expect the shape of the $(\operatorname{EM}(T))$ to not change by much qualitatively even its absolute values can change significantly.

For wide band instruments like XRT, the effect of the adopted chemical composition of the plasma on the temperature response is not as straightforward, as at different temperatures the relative contribution of the emission from different elements can change significantly. In Figure 13, we show the effect of the element abundances on the temperature response of the XRT filters used in this work. We consider the Feldman (1992) "coronal" abundances, and a set of abundances intermediate between coronal and photospheric (Grevesse \& Sauval 1998), i.e., with a FIP bias of 2 instead of 4 which is typically assumed for coronal plasma. For instance, for this set of abundances, Fe, Si, and Mg have abundances about half the values of Feldman (1992). Figure 13 shows that in the range $\log T[\mathrm{~K}] \sim 6.7-7.3$ the change in the responses is approximately linear with the abundances of these low-FIP elements which are dominating the emission in this high temperature range. At lower temperatures however the XRT filters are affected differently depending on the details of their response as a function of wavelength and therefore the importance of different lines.

We repeated our analysis using different sets of abundances, and in particular we derived the $(\operatorname{EM}(T))$ using this set of intermediate abundances described here above, and the set of photospheric abundances of Grevesse \& Sauval (1998). The results we obtained are shown in Figure 14, also compared to the $(\operatorname{EM}(T))$ derived with Feldman abundances. The curves have very similar shape and are overall compatible within the uncertainties. However, it is clear that, as expected in the light of the above discussions, the $(\operatorname{EM}(T))$ obtained for intermediate abundances (which are lower than the "coronal" abundances) is systematically larger than the one found for "coronal" abundances, and the 

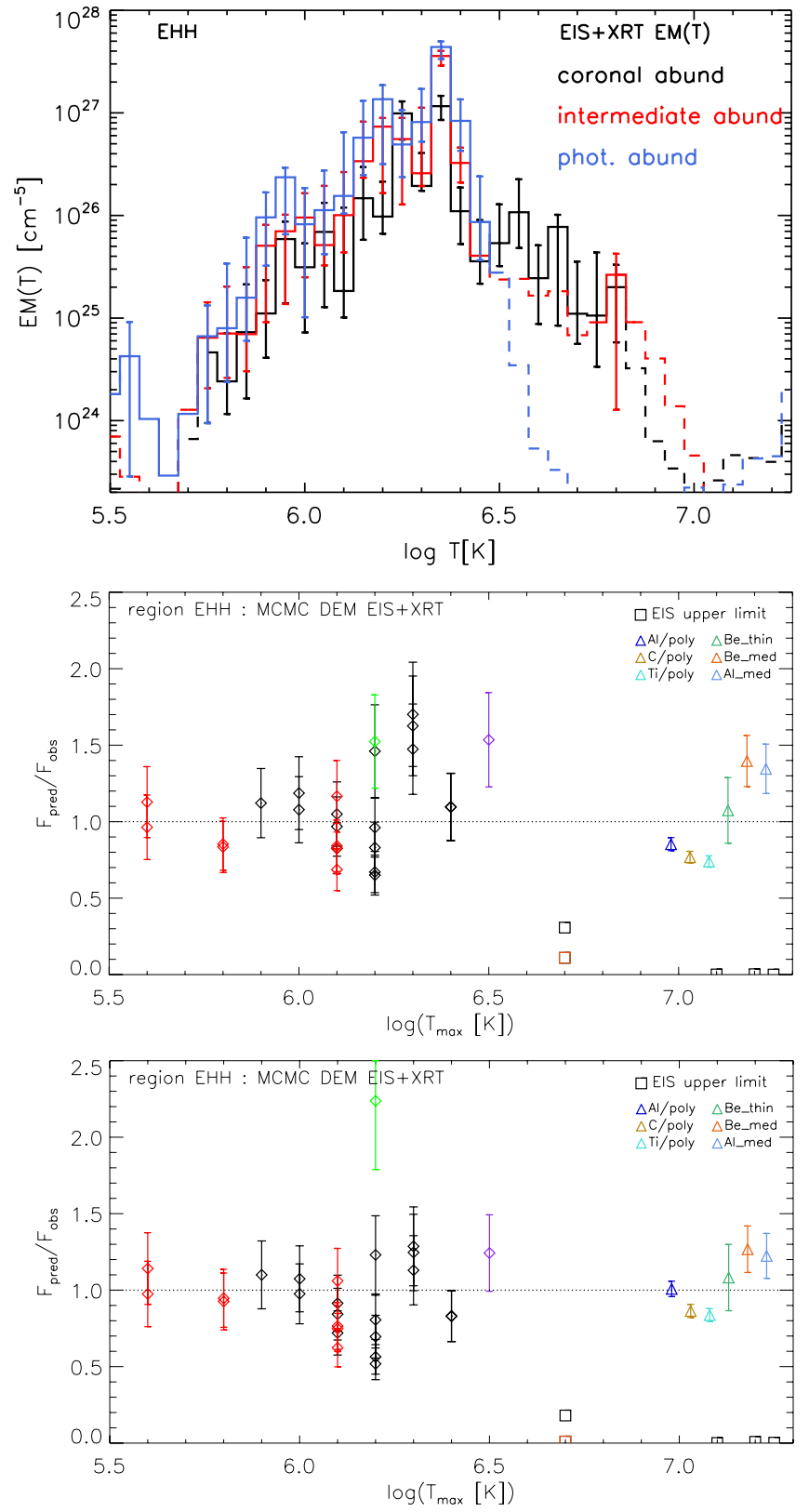

Figure 14. Top panel: comparison of emission measure distributions derived with the MCMC method using simultaneously EIS and XRT fluxes, and three different sets of abundances: coronal abundances (Feldman 1992; black line), intermediate abundances (red line; see the text for definition of intermediate abundances), and photospheric abundances (Grevesse \& Sauval 1998; blue line). For intermediate abundances, for which $\mathrm{Fe}$ and $\mathrm{Si}$ have roughly half the abundance as in the coronal set, the $(\operatorname{EM}(T))$ accordingly increases; the $(\operatorname{EM}(T))$ increases even further for photospheric abundances. Middle and bottom panels: comparison of measured EIS and XRT fluxes with flux values predicted by the emission measure distribution derived with the MCMC method (XRT+EIS) and (a) intermediate abundances (middle), or (b) photospheric abundances (bottom; Grevesse \& Sauval 1998).

$(\operatorname{EM}(T))$ derived for the photospheric abundance cases are even larger. In the middle panel of Figure 14, we show the comparison of the observed fluxes with the predictions using the $(\operatorname{EM}(T))$ derived for intermediate abundances. Comparing these findings with the analogous results of Figure 12 we note that although the overall results are not changed dramatically, the fluxes predicted for the XRT thin filters are closer to the observations when using intermediate abundances. The increases by about $20 \%-35 \%$ are compatible with expectations: the predicted fluxes should rise accordingly to the increase of the cool component of the $(\operatorname{EM}(T))$ but be reduced by a factor corresponding to the decrease of the XRT response (see Figure 13). The analogous plot for the case of photospheric abundances (bottom panel of Figure 14) shows that using these abundance values the agreement between EIS and XRT improves even further.

The values assumed for the element abundances can in principle be checked with the EIS spectra. As discussed above, the DEM obtained from EIS has been determined using almost exclusively lines from low-FIP ions, whose coronal abundances are enhanced by a factor of $\sim 3-4$ over the photospheric values. Therefore, any change in the actual abundance of the low-FIP elements from the assumed value will result in a systematic shift between the observed intensities of the lines from $\mathrm{S} x$ to $\mathrm{S}$ XIII, whose coronal abundance is expected to be close to the photospheric one, and their values predicted with the DEM. There are ten $\mathrm{S}$ lines in the EIS spectra, emitted by $\mathrm{S} x$ to $\mathrm{S}$ XIII, and we have compared their predicted and observed intensities to check the corrections to the coronal abundances. We found no unambiguous evidence of systematic differences in the observed to predicted intensity ratios. Moreover, recent estimates of the S absolute abundance have been revised by Lodders (2003) and by Caffau \& Ludwig (2007), who decreased them by a factor of 1.5 from the Grevesse \& Sauval (1998) value. Since this change is of the same order of the revision of the low-FIP element abundances we propose, we feel that the uncertainties in the S-predicted line intensities are so large that these ions cannot be used to confirm the abundance change made necessary by the XRT channels in this work. $\mathrm{S}$ is in any case a borderline element for FIP effect studies, and abundances of other lowFIP elements are difficult to determine. While oxygen lines of $\mathrm{O}$ IV,V,VI are present in the EIS wavelength range, they are formed at low temperature $(\log (T(\mathrm{~K})) \lesssim 5.5)$ with respect to the hotter coronal EIS lines considered in our active region study. In that temperature range the thermal distribution is not well constrained and therefore the abundances cannot be accurately determined.

While the results shown in Figure 14 seem to suggest that the photospheric abundances might be a more appropriate choice for the active region we studied here, we note that the data we have used do not allow us to determine the abundances and therefore do not allow us to disentangle the abundance effects and cross-calibration issues. We also note that this active region was already several days old at the time of the observation, and therefore it is reasonable to expect some significant effect of chemical fractionation to have occurred producing departures from the photospheric composition (see, e.g., Widing \& Feldman 2001; however, see also Del Zanna 2003).

We have presented the complete analysis carried out for one of the selected regions, providing insights into the thermal distribution of the plasma, limitations of the methods, and crosscalibration of the Hinode XRT and EIS. Before discussing in details these findings in Section 4, we briefly present and discuss the analogous results found for the other two selected regions, $\mathrm{EC}$ and $\mathrm{EH}$, comparing them with the results obtained for region EHH.

Carrying out the same analysis described above (Sections 3.1-3.3) on the other two regions, we find results qualitatively similar to our findings for region EHH discussed above, in particular in terms of the comparison between different analysis methods and systematic discrepancies between XRT and EIS. 

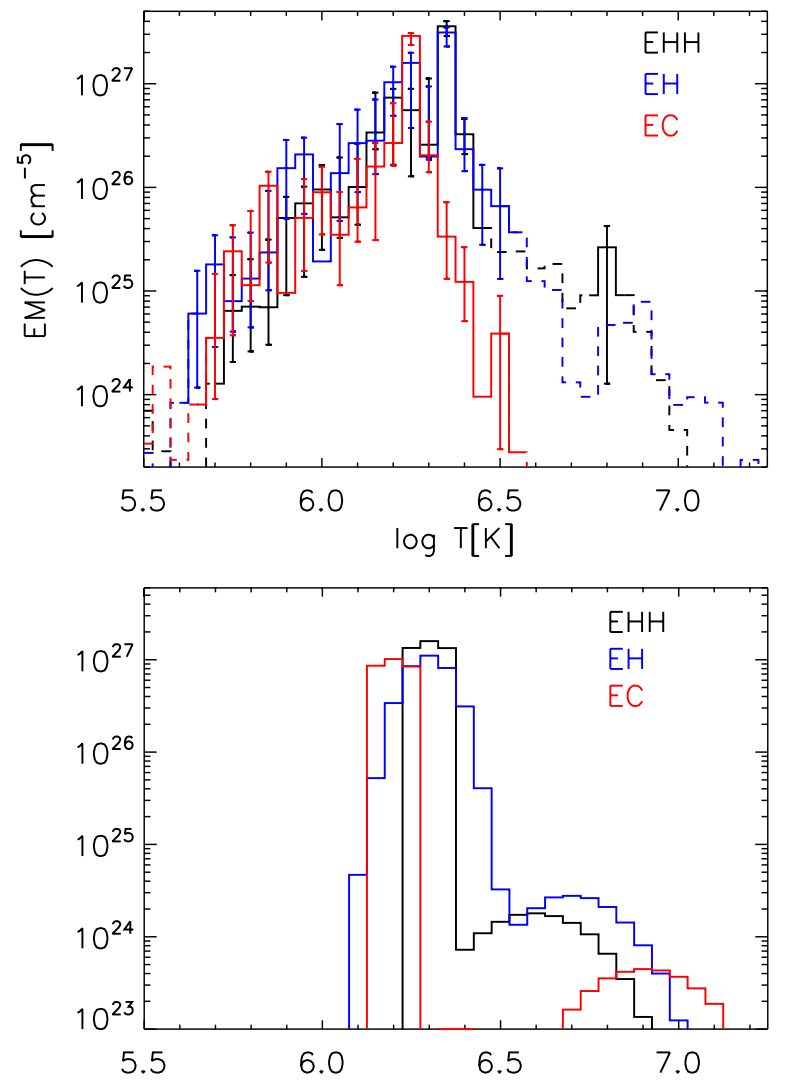

Figure 15. Emission measure distributions for the three selected regions: EC (red line), EH (blue line), and EHH (black line). Top: $(\operatorname{EM}(T))$ curves derived with MCMC method, using EIS and XRT fluxes. Bottom: $(\operatorname{EM}(T))$ derived from XRT data through the forward fitting $2 \mathrm{~T}$ method (see Section 3.2 for details), using photospheric abundances.

In Figure 15, we show the emission measure distributions obtained for the three regions, using the MCMC algorithm applied to XRT and EIS data together (top panel), or forward fitting the XRT observations using two temperature components (bottom panel). We note that despite the forward fitting $2 T$ model adopts considerable simplifying assumptions for the underlying emission measure distribution, the results we obtain with this method are qualitatively in good agreement with the MCMC approach which does not impose constraints on the shape of the DEM. On the other hand, we note that the forward fitting $2 T$ model has the advantage of also taking into account the spatial information, while the MCMC method only uses the information contained in the integrated fluxes in each passband. The two hotter regions, $\mathrm{EH}$ and $\mathrm{EHH}$, have very similar underlying $(\operatorname{EM}(T))$ : their cool components have analogous peak temperature $(\log T(\mathrm{~K}) \sim 6.2-6.3)$ and amounts of plasma, whereas the hot component of $\mathrm{EHH}$ is of weight comparable to the high temperature component of EH but it appears shifted toward slightly higher temperatures. For region $\mathrm{EC}$, the cool component is characterized by a peak temperature slightly cooler than the other two regions, and in particular its $(\operatorname{EM}(T))$ falls off faster on the high temperature side of the cool peak; the hot component of EC is much weaker than the other two components, if at all present.

\section{DISCUSSION}

We have presented the results of a detailed analysis of Hinode XRT and EIS observations of a non-flaring active region to diagnose the temperature distribution of the coronal plasma. In this work, we carried out independent temperature analysis from measurements of either instrument, and use the derived thermal distributions to make prediction for the fluxes observed with the other instrument and then compare them with actual measurements. Then, we combine the information from both instruments and compare the results with the findings based on XRT or EIS data only. This approach allowed us to explore the limitations of the data and analysis methods in providing constraints to the plasma temperature distribution, and investigate the cross-calibration of the two Hinode instruments. With respect to previous works using both XRT and EIS data to study the thermal properties of the coronal plasma (e.g., Landi et al. 2010), in this work we provide a quantitative cross-check between the diagnostics of the two instruments.

When using the two data sets separately the derived $(\operatorname{EM}(T))$ curves have overall similar width and peak temperature. However, the emission measure derived from EIS is systematically smaller, by a factor $\sim 2-3$, than the emission measure derived from XRT fluxes for each of the three studied regions. We note that even if the uncertainties in the derived $(\operatorname{EM}(T))$ are rather large the systematic discrepancy appears to be significant. We find that the extent of the discrepancy between XRT and EIS depends in part on the assumptions made for the chemical composition of the X-ray emitting plasma: it can be mitigated by assuming abundances intermediate between the typical "coronal" abundances (Feldman 1992) and the photospheric values (Grevesse \& Sauval 1998), and it almost disappears when using photospheric abundances. This interesting finding encourages further investigations to search for similar evidence in other active regions, in an active region at different epochs, and in different kinds of coronal structures (quiet Sun, bright points, coronal holes). While our study provides no robust constraints on the element abundances, we stress the importance of the assumed values in the analysis of Hinode observations. The detailed $(\mathrm{EM}(T))$ reconstruction and the instrument cross-calibration are significantly affected by the assumed abundances: EIS spectra allow the determination of "abundance independent" $(\operatorname{EM}(T))$, e.g., using exclusively Fe lines (Watanabe et al. 2007), but its absolute values depend on the abundances; the XRT passbands include significant contribution of several elements, both high-FIP and low-FIP, and therefore the dependence of the $(\mathrm{EM}(T))$ on abundances is more complicated and temperature dependent.

For the analysis of XRT data, we use two different methods for deriving the emission measure distribution. We find that the MCMC method yields results in good qualitative agreement with the forward fitting $2 T$ method which makes rather simplifying assumptions on the emission measure distribution but has the advantage of making use also of the spatial information. This finding lends further confidence to the results obtained previously by applying this method to the study of XRT observations of another active region (Reale et al. 2009b).

We find that the EIS spectra allow an accurate determination of the cool $(\log T(\mathrm{~K}) \lesssim 6.5)$, most prominent, component, but when used alone the EIS data are unable to constrain the hotter emission due to the lack of strong hot lines in the spectra. The combination with XRT data provide much tighter constraints on the emission measure distribution on a wider temperature range. Our analysis shows that the studied active region is characterized by bulk plasma temperatures of $\sim 2 \mathrm{MK}$, which are rather low for active region plasma. The $(\operatorname{EM}(T))$ of the three selected regions are very similar for $\log T(\mathrm{~K}) \lesssim 6.2$, but for the two 
hotter regions, $\mathrm{EH}$ and $\mathrm{EHH}$, the cool component is broader and with a peak shifted toward higher $T$. The high temperature tail of the emission measure distribution is not strongly constrained by the data. However, the $(\operatorname{EM}(T))$ derived at least for the two hotter regions, $\mathrm{EH}$ and $\mathrm{EHH}$, suggests the presence of a hotter component $(\log T(\mathrm{~K}) \gtrsim 6.5)$ about two orders of magnitude weaker than the dominant cool component in good agreement with the findings of Reale et al. (2009b) for another, hotter, active region.

\section{CONCLUSIONS}

We analyzed Hinode XRT multi-filter data and EIS spectral observations of a non-flaring active region to study the temperature distribution of coronal plasma, $(\operatorname{EM}(T))$, and to carry out a detailed investigation of the cross-calibration of the two instruments. We selected three subareas of the active region, and for each of them we derived the emission measure distribution $(\mathrm{EM}(T))$ by (1) using EIS measured line fluxes, (2) using XRT fluxes in six of the instrument's filters, and (3) combining the data sets for the two instruments.

We find a good consistency in the qualitative characteristicspeak temperature and width of dominant temperature component - of the $(\operatorname{EM}(T))$ derived with different methods. However, the emission measure distributions derived by the two instruments XRT and EIS indicate a systematic discrepancy between the two instruments, with EIS data yielding $(\operatorname{EM}(T))$ consistently smaller, by about a factor two, than the $(\operatorname{EM}(T))$ compatible with XRT data. We discuss the possible origin of the disagreement and find that the assumptions for the element abundances significantly influence the plasma temperature diagnostics. In particular, we find that a chemical composition intermediate between the usually adopted coronal abundances by Feldman (1992) and the solar photospheric abundances improves the comparison between the results obtained with the two instruments. When adopting photospheric abundances the discrepancy between EIS and XRT decreases further. However, we note that it seems unlikely that the observed plasma, in an active region which is not newly emerged, has photospheric composition. Furthermore, the used data do not allow a definite determination of the abundances, and therefore do not allow us to robustly assess of the cross-calibration of the instruments.

One of the main aims of this work was to exploit the complementary diagnostics for the X-ray emitting plasma provided by XRT and EIS, to investigate the presence of hot plasma in non-flaring regions, and to test nanoflare heating models. We find that the derived $(\operatorname{EM}(T))$ are characterized by an expected dominant cool component (typically $\log T(\mathrm{~K}) \sim 6.3$ ), and a much weaker amount of plasma at higher temperature. While the amount of hot plasma is in general in agreement with recent findings for other non-flaring active regions, and it is compatible with expectations from nanoflare models, we find that within the uncertainties these results are not conclusive.

We thank P. Grigis and J. Drake for useful discussions. Hinode is a Japanese mission developed and launched by ISAS/ JAXA, with NAOJ as domestic partner and NASA and STFC (UK) as international partners. It is operated by these agencies in co-operation with ESA and the NSC (Norway). P.T. and E.E.D. were supported by NASA contract NNM07AB07C to the Smithsonian Astrophysical Observatory. F.R. acknowledges support from Italian Ministero dell'Università e Ricerca and
Agenzia Spaziale Italiana (ASI), contract I/015/07/0. The work of Enrico Landi is supported by NNH06CD24C, NNH09AL49I, and other NASA grants.

\section{REFERENCES}

Aschwanden, M. J., Nightingale, R. W., \& Alexander, D. 2000, ApJ, 541, 1059

Brooks, D. H., Warren, H. P., Williams, D. R., \& Watanabe, T. 2009, ApJ, 705, 1522

Brosius, J. W., Davila, J. M., Thomas, R. J., \& Monsignori-Fossi, B. C. 1996, ApJS, 106, 143

Bryans, P., Landi, E., \& Savin, D. W. 2009, ApJ, 691, 1540

Caffau, E., \& Ludwig, H. 2007, A\&A, 467, L11

Cargill, P. J. 1994, ApJ, 422, 381

Cargill, P. J., \& Klimchuk, J. A. 1997, ApJ, 478, 799

Craig, I. J. D., \& Brown, J. C. 1976, A\&A, 49, 239

Culhane, J. L., et al. 2007, Sol. Phys., 243, 19

Del Zanna, G. 2003, A\&A, 406, L5

Del Zanna, G., \& Mason, H. E. 2003, A\&A, 406, 1089

Dere, K. P., Landi, E., Mason, H. E., Monsignori Fossi, B. C., \& Young, P. R. 1997, A\&AS, 125, 149

Dere, K. P., Landi, E., Young, P. R., Del Zanna, G., Landini, M., \& Mason, H E. 2009, A\&A, 498, 915

Doschek, G. A., Mariska, J. T., Warren, H. P., Culhane, L., Watanabe, T., Young, P. R., Mason, H. E., \& Dere, K. P. 2007, PASJ, 59, 707

Feldman, U. 1992, Phys. Scr, 46, 202

Golub, L., et al. 2007, Sol. Phys., 243, 63

Grevesse, N., \& Sauval, A. J. 1998, Space Sci. Rev., 85, 161

Judge, P. G. 2010, ApJ, 708, 1238

Judge, P. G., Hubeny, V., \& Brown, J. C. 1997, ApJ, 475, 275

Kashyap, V., \& Drake, J. J. 1998, ApJ, 503, 450

Kashyap, V., \& Drake, J. J. 2000, Bull. Astron. Soc. India, 28, 475

Klimchuk, J. A. 2006, Sol. Phys., 234, 41

Kosugi, T., et al. 2007, Sol. Phys., 243, 3

Landi, E., Feldman, U., \& Dere, K. P. 2002, ApJS, 139, 281

Landi, E., \& Landini, M. 1998, A\&A, 340, 265

Landi, E., Miralles, M. P., Curdt, W., \& Hara, H. 2009, ApJ, 695, 221

Landi, E., Raymond, J. C., Miralles, M. P., \& Hara, H. 2010, ApJ, 711, 75

Lang, J., et al. 2006, Appl. Opt., 45, 8689

Lodders, K. 2003, ApJ, 591, 1220

McTiernan, J. M. 2009, ApJ, 697, 94

Meyer, J. 1985, ApJS, 57, 173

Narukage, N., et al. 2010, Sol. Phys., in press (arXiv:1011.2867)

O’Dwyer, B., Del Zanna, G., Mason, H. E., Sterling, A. C., Tripathi, D., \& Young, P. R. 2011, A\&A, 525, 137

Parenti, S., Buchlin, E., Cargill, P. J., Galtier, S., \& Vial, J. 2006, ApJ, 651, 1219

Parker, E. N. 1972, ApJ, 174, 499

Parker, E. N. 1988, ApJ, 330, 474

Patsourakos, S., \& Klimchuk, J. A. 2009, ApJ, 696, 760

Reale, F., McTiernan, J. M., \& Testa, P. 2009a, ApJ, 704, L58

Reale, F., Testa, P., Klimchuk, J. A., \& Parenti, S. 2009b, ApJ, 698, 756

Reale, F., et al. 2007, Science, 318, 1582

Schmelz, J. T., Saar, S. H., DeLuca, E. E., Golub, L., Kashyap, V. L., Weber, M. A., \& Klimchuk, J. A. 2009a, ApJ, 693, L131

Schmelz, J. T., et al. 2009b, ApJ, 704, 863

Shestov, S. V., Kuzin, S. V., Urnov, A. M., Ul'Yanov, A. S., \& Bogachev, S. A. 2010, Astron. Lett., 36, 44

Sylwester, B., Sylwester, J., \& Phillips, K. J. H. 2010, A\&A, 514, A82

Testa, P., Peres, G., Reale, F., \& Orlando, S. 2002, ApJ, 580, 1159

Tripathi, D., Mason, H. E., Del Zanna, G., \& Young, P. R. 2010, A\&A, 518, A42

Warren, H. P., \& Brooks, D. H. 2009, ApJ, 700, 762

Warren, H. P., Kim, D. M., DeGiorgi, A. M., \& Ugarte-Urra, I. 2010, ApJ, 713, 1095

Warren, H. P., Ugarte-Urra, I., Doschek, G. A., Brooks, D. H., \& Williams, D. R. 2008, ApJ, 686, L131

Warren, H. P., Winebarger, A. R., \& Mariska, J. T. 2003, ApJ, 593, 1174

Watanabe, T., Hara, H., Culhane, L., Harra, L. K., Doschek, G. A., Mariska, J. T., \& Young, P. R. 2007, PASJ, 59, 669

Watanabe, T., et al. 2009, ApJ, 692, 1294

Widing, K. G., \& Feldman, U. 2001, ApJ, 555, 426

Young, P. R., Landi, E., \& Thomas, R. J. 1998, A\&A, 329, 291

Young, P. R., Watanabe, T., Hara, H., \& Mariska, J. T. 2009, A\&A, 495, 587

Young, P. R., et al. 2007, PASJ, 59, 857 